\title{
Genetic Effects of Trabecular Titanium on MG-63 Cell Line: A Genetic Profiling Evaluation
}

\section{Sollazzo Vincenzo, ${ }^{1}$ Massari Leo, ${ }^{1}$ Pezzetti Furio, ${ }^{2}$ Girardi Ambra, ${ }^{2}$ Farinella Francesca, ${ }^{3}$ Lorusso Vincenzo, ${ }^{1}$ Burelli Silvia, ${ }^{4}$ Bloch Hans Rudolf, ${ }^{4}$ and Carinci Francesco ${ }^{3}$}

${ }^{1}$ Orthopaedic Institute, University of Ferrara, Corso Giovecca 203, 44100 Ferrara, Italy

${ }^{2}$ Department of Histology, Embryology and Applied Biology, University of Bologna, 40126 Bologna, Italy

${ }^{3}$ Department of Maxillofacial Surgery, University of Ferrara, 44121 Ferrara, Italy

${ }^{4}$ Lima Corporate, 30030 S. Daniele del Friuli, Italy

Correspondence should be addressed to Massari Leo, msl@unife.it

Received 13 April 2011; Accepted 9 May 2011

Academic Editors: E. Burkel and K. Kusabiraki

Copyright ( $) 2011$ Sollazzo Vincenzo et al. This is an open access article distributed under the Creative Commons Attribution License, which permits unrestricted use, distribution, and reproduction in any medium, provided the original work is properly cited.

Pure titanium and titanium alloys are materials widely used in orthopaedics because of their mechanical properties, chemical stability, and biocompatibility. Recently, a new highly porous titanium biomaterial named Trabecular Titanium (TT) has been developed. In this in vitro study, we tested the genetic effects of TT on osteoblast-like cells (MG63) using DNA microarrays technology: cDNA microarrays provides the ability to comparatively analyze mRNA expression of thousands of genes in parallel thus showing activated and repressed genes by the presence of the TT. Several genes that were significantly up- or downregulated have been identified. Globally, it was demonstrated that TT stimulates osteoblasts proliferation and differentiation, and reduces apoptosis. Therefore, all these effects can contribute to improve the osseointegration of this material. These results encourage the clinical application of Trabecular Titanium to prosthetic devices.

\section{Introduction}

Several features of the implant surface such as composition, topography, roughness, and energy level play a relevant role in implant osseointegration $[1,2]$. Little is known about the structural and chemical surface properties that may influence biological responses [3]. Pure titanium and titanium alloys are materials widely used in orthopaedics and dental surgery because of their mechanical properties, chemical stability, and biocompatibility $[4,5]$. In fact, titanium is used to manufacture joint prostheses for partial and total joint replacement. Moreover, titanium is also used to produce plates and screws for the osteosynthesis of fractures and for dental implants $[6,7]$. Surface roughness has been demonstrated to have positive effects on adsorption of molecules, local factor production, and cells proliferation and differentiation [8-10]. Conventional orthopaedic implants are composed of stainless steel, cobalt-chromium $(\mathrm{CoCr})$, and titanium alloys [11]. Titanium is the most commonly used metal for cementless implants because of its excellent biocompatibility, ready availability, and high strength, as regards to its low specific gravity [12]. Although good clinical results have been shown, traditional porous materials have several inherent limitations, such as low volumetric porosity, relatively high modulus of elasticity, low frictional characteristics, and availability as a coating only. With the aim of surmounting these limits, improving the potentiality of osteointegration and, consequently the stability of prosthetic implants, a multiplanar, hexagonal, highly porous biomaterial named Trabecular Titanium (TT) which imitates the cell structure of the cancellous bone has been developed. The morphology and dimension of the open pores have been developed to induce osseointegration. The resistance of this material is higher than the cancellous bone, and the low rigidity has been studied to foster the transmission of physiological loads from implant to bone.

To thoroughly investigate the mechanism of interaction between TT and osteoblasts, we attempted to analyse it 


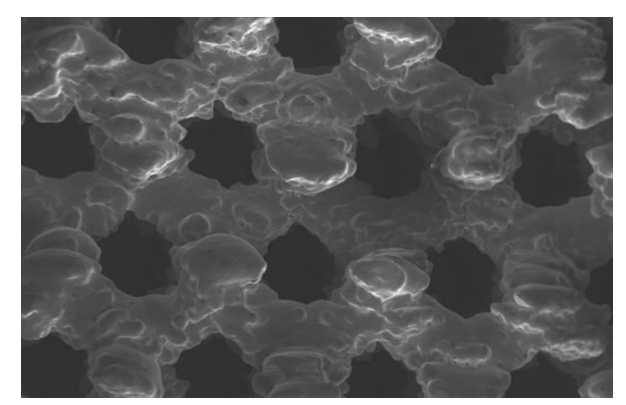

Figure 1: Trabecular Titanium three-dimensional highly porous structure.

genetically by using microarray techniques. In previous in vitro study, a preliminary study was conducted with MG63 cultured on TT [13].

Here a larger study was conducted to verify osteoblastlike cell line (MG63) behaviour in cultures grown on disks of this innovative three-dimensional porous biomaterial by using a microarray of slides containing 20,000 different oligonucleotides.

\section{Material and Methods}

2.1. Cell Culture. Osteoblast-like cells (MG63) were cultured in sterile Falcon wells (Becton Dickinson, New Jersey, USA) containing Eagle's minimum essential medium (MEM) supplemented with 10\% foetal bovine serum (FBS) (Sigma, Chemical Co., St. Louis, Mo, USA) and antibiotics (penicillin $100 \mathrm{U} / \mathrm{mL}$ and streptomycin $100 \mu \mathrm{g} / \mathrm{mL}$-Sigma, Chemical Co, St. Louis, Mo, USA). Cultures were maintained in a $5 \%$ $\mathrm{CO}_{2}$ humidified atmosphere at $37^{\circ} \mathrm{C}$.

MG63 cells were collected and seeded at a density of $1 \times 10^{5}$ cells $/ \mathrm{mL}$ into $9 \mathrm{~cm}^{2}(3 \mathrm{~mL})$ wells by using $0.1 \%$ trypsin, $0.02 \%$ EDTA in $\mathrm{Ca}^{2+}$ - and Mg-free Eagle's buffer for cell release. One set of wells contained sterile disks of Trabecular Titanium (Lima Corporate, Villanova di San Daniele, Udine, Italy)., Ti6Al4V, $30 \mathrm{~mm}$ in diameter and $5 \mathrm{~mm}$ in height, covering the same area as the reference wells. Two structures with two different open porosities have been tested: one has a regular three-dimensional hexagon unit cell with a mean pore dimension of $644 \mu \mathrm{m}$ (TT600), whereas the other has a mean pore dimension of $1238 \mu \mathrm{m}$ (Figure 1). The control wells contained the same cells seeded on conventional machined titanium (Lima Corporate, Villanova di San Daniele, Udine, Italy) and the same medium. After 24 hours, when the cultures were subconfluent, the cells were processed for RNA extraction.

2.2. DNA Microarray Screening and Data Analysis. Total RNA was extracted from cells by using a Total RNA Isolation Kit (Nucleospin RNA II Isolation Kit). Eight hundred nanograms of total RNA were used to synthesize cDNA, using Amersham CyScribe Post-Labelling Kit (GE Healthcare Little Chalfont, UK). Monoreactive Cy3 and Cy5 esters (present in the same kit) were used for indirect cDNA labelling. RNA extracted from untreated cells was labelled with Cy3 and used as a control against the Cy5labelled treated cDNA in the first experiment and then switched. For 19008 human DNA microarray slides (Corning UltraGAP, Ontario, Canada), $100 \mu \mathrm{L}$ of the sample and control cDNAs in DIG Easy hybridisation solution (Roche, Basel, Switzerland) was used in a sandwich hybridisation of the two slides constituting the 20000 set, which were incubated at $37^{\circ} \mathrm{C}$ overnight. Washing was performed 3 times for 10 minutes with $1 \times$ saline sodium citrate (SSC), $0.1 \%$ sodium dodecyl sulphate (SDS) at $42^{\circ} \mathrm{C}$, and 3 times for 5 minutes with $0.1 \times$ SSC at room temperature. Slides were dried by centrifugation for 2 minutes at $2000 \mathrm{rpm}$.

After washing and drying, the hybridised slides were scanned using a GenePix 4000A scanner (Axon Instruments, Foster City, Calif, USA). To correctly evaluate the samples, scanning was performed to obtain a similar level of emissions for the two dyes. Data normalization was performed by the free software tool BRB-Array Tools (http://linus.nci.nih.gov/ BRB-ArrayTools.html).

Genes differentially expressed in MG63 cultures with respect to MG63-PT growth were identified using the Significance Analysis of Microarrays (SAM) procedure. SAM uses an algorithm based on the Student's $t$-test and estimates any false discovery rate (FDR), which is the percentage of genes falsely reported as showing statistically significant differential expression. A false discovery rate of 0 was used as the threshold identifying differentially expressed genes.

2.3. RNA Processing. Reverse transcription to cDNA was performed directly from cultured cell lysate using the TaqMAN Gene Expression Cells-to-Ct Kit (Ambion Inc., Austin, Tex, USA), following manufacturer's instructions. Briefly, cultured cells were lysed with lysis buffer and RNA released in this solution. Cell lysate were reverse transcribed to cDNA using the RT Enzyme Mix and appropriate RT buffer (Ambion Inc., Austin, Tex, USA).

Finally the cDNA was amplified by real-time PCR using the included TaqMan Gene Expression Master Mix and the specific assay designed for the investigated genes.

2.4. Real Time PCR. Expression was quantified using real time RT-PCR. The gene expression levels were normalized to the expression of the housekeeping gene RPL13A and were expressed as fold changes relative to the expression of the untreated cells. Quantification was done with the delta/delta calculation method.

Forward and reverse primers and probes for the selected genes were designed using primer express software (Applied Biosystems, Foster City, Calif, USA) and are RHOU and FBF1.

All PCR reactions were performed in a $20 \mu \mathrm{L}$ volume using the ABI PRISM 7500 (Applied Biosystems, Foster City, Calif, USA). Each reaction contained $10 \mu \mathrm{L} 2 \mathrm{X}$ TaqMan universal PCR master mix (Applied Biosystems, Foster City, Calif, USA), $400 \mathrm{nM}$ concentration of each primer and $200 \mathrm{nM}$ of the probe, and cDNA. The amplification profile was initiated by 10 -minute incubation at $95^{\circ} \mathrm{C}$, followed by two-step amplification of 15 seconds at $95^{\circ} \mathrm{C}$ and 60 
TABLE 1: Upregulated genes. Only the 10 more expressed genes are reported. The remaining are available on request.

\begin{tabular}{lclcc}
\hline GenBank & Symbol & Name & Cytoband & Score $(d)$ \\
\hline BE895065 & SLC39A9 & Solute carrier family 39 (zinc transporter), member 9 & $14 \mathrm{q} 24.1$ & 2,24 \\
AA011707 & RHOU & Ras homolog gene family, member U & 1q42.11-q42.3 & 1,90 \\
H17390 & ZDHHC18 & Zinc finger, DHHC-type containing 18 & 1p36.11 & 1,82 \\
AA001671 & CREB3L2 & CAMP responsive element binding protein 3-like 2 & $7 \mathrm{q} 34$ & 1,57 \\
H06956 & PLP1 & Proteolipid protein 1 & Xq22 & 1,55 \\
AA010246 & TTC17 & Tetratricopeptide repeat domain 17 & 11p12-p11.2 & 1,51 \\
H12716 & LRRFIP1 & Leucine rich repeat (in FLII) interacting protein 1 & $2 \mathrm{q} 37.3$ & 1,46 \\
W39189 & PPP1R10 & Protein phosphatase 1, regulatory (inhibitor) subunit 10 & $6 \mathrm{p} 21.3$ & 1,43 \\
N43819 & IQCB1 & IQ motif containing B1 & 3q13.33-3q21.1 & 1,36 \\
N79649 & MEMO1 & Mediator of cell motility 1 & 2p22-p21 & 1,33 \\
\hline
\end{tabular}

seconds at $60^{\circ} \mathrm{C}$ for 40 cycles. All experiments were performed including nontemplate controls to exclude reagents contamination.

\section{Results}

The genes differentially expressed in cells cultured on TT are reported in Tables 1 and 2, whereas in Figure 2 is reported the SAM plot. We briefly analysed some of those with well known functions.

3.1. Upregulated Genes. Interesting genes with elevated expression are genes related to cell differentiation, like parathyroid hormone-like hormone (PTHLH), which is a member of the parathyroid hormone family. This hormone regulates endochondral bone development. CREB3L2 (cAMP responsive element binding protein 3 -like 2) is upregulated as well. This gene encodes for a transcription factor, a c-AMP response element binding, that induces proliferation and differentiation processes.

Some genes acting at a transcriptional level are over expressed like ZDHHC18 (zinc finger, DHHC-type containing 18), which encodes a zinc finger protein.

3.2. Downregulated Genes. Some downregulated genes are related with signal transduction, like FYB (FYN binding protein (FYB-120/130), also known as ADAP. This gene encodes an adhesion and degranulation promoting adaptor protein for ADAP. ADAP possesses a number of domains predicted to mediate protein-protein interaction and is known to play an important role in "inside out" signalling leading to the activation of integrins and to enhanced cellular adhesion to integrin ligands.

Some genes related to regulation of cellular processes are downregulated. IKBKB (inhibitor of kappa light polypeptide gene enhancer in B-cells, kinase beta) encodes for one of the two catalytic subunits of the IkappaB kinase (IKK) complex that mediate NF-kappaB activation, IKKalpha and IKKbeta. The IKKbeta subunit is a transducer of signals from the receptor activator of NF-kappabeta (RANK) to NFkappabeta [14].

Several interesting downregulated genes encode for growth factors. FGFR2 (fibroblast growth factor receptor 2)

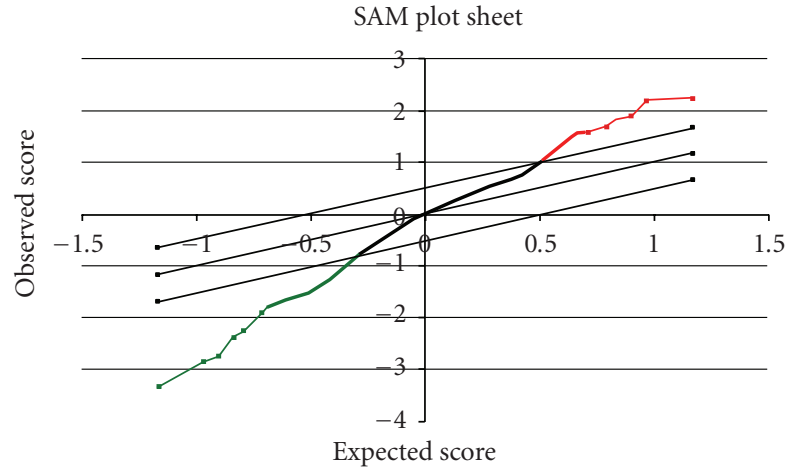

Figure 2: Statistical analysis of microarray (SAM) plot of MG63 cultured on PT disks versus control. Expected differentially expressed genes are reported along the $x$ axis, whereas observed differentially expressed genes are along the $y$ axis. Downregulated genes (green dots) are located in the lower left side of the diagram; upregulated genes (red dots) are in the upper right side; genes with different expression but statistically not significant are depicted in black. Parallel lines drawn from lower-left to upper-right squares are the cut-off limits. The solid line indicates the equal value of observed and expected differentially expressed genes.

encodes a protein that is a member of the fibroblast growth factor receptor family. The osteoblast response to an increase of FGF expression is inhibition of the differentiation process [15]. IGFBP5 (insulin-like growth factor-binding protein 5) gene encodes for insulin-like growth factor-binding protein 5. IGFBP-5 is the most abundant IGFBP stored in human bones, because it is the only IGFBP that binds avidly to hydroxyapatite [16].

Some downregulated genes are related to the degradation of extracellular matrix, specifically ADAM10 (ADAM metallopeptidase domain 10) and ADAM23 (ADAM metallopeptidase domain 23). Those genes encode a protein of the ADAM (a disintegrin and metalloprotease domain) family. Members of this family are membrane-anchored proteins that are implicated in a variety of biological processes involving cellcell and cell-matrix interactions. ADAM proteins contain a disintegrin and a MMP domain, which have the dual function of cleavage/release of cell-surface proteins and remodelling of the extracellular matrix [17]. 
TABle 2: Downregulated genes. Only the 10 less expressed genes are reported. The remaining are available on request.

\begin{tabular}{|c|c|c|c|c|}
\hline GenBank & Symbol & Name & Cytoband & Score $(d)$ \\
\hline T80466 & CA5B & Carbonic anhydrase VB, mitochondrial & Xp21.1 & $-2,86$ \\
\hline R48853 & SNX24 & Sorting nexin 24 & $5 \mathrm{q} 23.2$ & $-1,74$ \\
\hline N52833 & FAM27E3 & Family with sequence similarity 27 , member E3 & $9 q 12$ & $-1,74$ \\
\hline R61149 & C1orf173 & Chromosome 1 open reading frame 173 & $1 \mathrm{p} 31.1$ & $-1,72$ \\
\hline AA010034 & FBF1 & Fas (TNFRSF6) binding factor 1 & $17 \mathrm{q} 25.1$ & $-1,70$ \\
\hline BG939191 & DNAJA2 & DnaJ (Hsp40) homolog, subfamily A, member 2 & $16 \mathrm{q} 12.1$ & $-1,69$ \\
\hline AA150120 & RPS14 & Ribosomal protein S14 & $5 q 31-q 33$ & $-1,69$ \\
\hline BG354579 & CBX2 & Chromobox homolog 2 (Pc class homolog, Drosophila) & $17 q 25.3$ & $-1,68$ \\
\hline N28644 & FLJ22536 & Hypothetical locus LOC401237 & $6 \mathrm{p} 22.3$ & $-1,67$ \\
\hline $\mathrm{R} 21428$ & MAP2K1IP1 & Mitogen-activated protein kinase kinase 1 interacting protein 1 & $4 q 23$ & $-1,66$ \\
\hline
\end{tabular}

Other genes related to the immune system are downregulated. CXCL10 (chemokine (C-X-C motif) ligand 10). This gene encodes a chemokine of the CXC subfamily and ligand for the receptor CXCR3. CXCL10 is categorized functionally as a Th- 1 chemokine. Binding of this protein to CXCR3 results in pleiotropic effects, including stimulation of monocytes, natural killer and T-cell migration, and modulation of adhesion molecule expression [18]. S100A12 (S100 calcium binding protein A12) encodes for a protein that is a S100 family member, containing 2 EF-hand calcium-binding motifs. Serum levels of S100A12 correlate with disease activity in different arthropathies as juvenile idiopathic arthritis and reflect neutrophilic activation during synovial inflammation as RA $[19,20]$.

Some genes related with cellular biosynthetic processes are also downregulated. HSD11B2 (hydroxysteroid (11-beta) dehydrogenase 2) is a gene that encodes for hydroxysteroid (11-beta) dehydrogenase 2. This enzyme generates active cortisol from inactive cortisone (or prednisone) and regulates glucocorticoid action in vitro. Chronically high levels of glucocorticoids typically decrease bone mass in humans and animals, and inhibit markers of bone formation in organ and cell cultures [21].

Analysing the genetic portrait of Trabecular Titanium in two different porosities, we found no significant differences between the TT600 and TT1200 (data not shown).

RT-PCR results confirm the upregulation of RHOU and the downregulation of FBF1.

\section{Discussion}

Titanium and titanium alloys are widely used as implant materials due to their excellent biocompatibility and mechanical properties. The goal of prosthetic surgery is to have the best possible osseointegration to ensure stability and long-term survival rate of the implants [22]. Implant osseointegration at the tissue-prosthesis interface contributes to determining the success or failure of the device. Osteoblasts, growing on the implant surface, are responsible for the tissue response on the biomaterial surface [23], whose modality and quality depend on the surface morphology.

Generally, it is possible to distinguish the macro-, mini-, micro- and nanodesign of prostheses. The macrodesign is the shape of the prosthesis. The minidesign is related to the featured dimensions of the prosthesis, like indentations, threads and fins. The micro-design is the shape of the implant surface, due to surface treatments like machining, acid etching, and sand-blasting procedures that determine the surface roughness, characterised by a cellular dimension. Finally, the nanodesign is determined by the molecular composition of the surface. The conventional porous Titanium surface is rough, and it contains many pores and grooves of different sizes regularly distributed over the whole surface. The osteoblasts are sensitive to surface roughness and material composition. It was shown that osteoblast-like cells (MG63) grown on rough titanium surfaces exhibited a reduced cell proliferation rate but increased alkaline phosphatase-specific activity and osteocalcin production [9, $24,25]$. Titanium promotes protein adsorption, the crucial factor for the initial cell adhesion on artificial surfaces. Molecules involved in cell adhesion include extracellular matrix proteins, transmembrane receptors and intracellular cytoskeletal components [26].

In this study, we tested disks of Trabecular Titanium (TT) to determine the genetic effects on human osteoblastlike cells (osteosarcoma cell line MG-63) using microarray technology. Hybridisation of mRNA-derived probes to cDNA microarrays allowed us to perform systemic analysis of expression profiles for thousand of genes simultaneously and to provide primary information on transcriptional changes related to TT action. Since DNA microarray is a molecular technology that enables a complete large-scale gene expression analysis, spanning a significant fraction of the human genome gives a global view of the genetic effect of a factor on a cell system. In fact, DNA microarray is not only a quantitative analysis, but also a qualitative one, differentiating a single gene among a thousand sequences.

The exposure to the multiplanar, hexagonal, highly porous TT induced the upregulation of several genes related to cellular proliferation and differentiation via transcription, like CREB3L2 and PTHLH. The PTHLH upregulation may delay the closure of the growth plates and promote endochondral bone formation [27]. The upregulation of PTHLH can be interpreted as an induction of endochondral ossification leading to potential improvement of biocompatibility of the implant. Moreover, the upregulation of 
CREB3L2 can induce proliferation and differentiation of osteoblasts.

Trabecular Titanium induced the downregulation of several genes involved in bone resorption, in the regulation of matrix degradation and immunity. In detail, it induced the downregulation of $\mathrm{C}-\mathrm{Src}$, which plays a crucial role in osteoclastogenesis [28, 29]. IKKbeta expression also resulted in downregulation. It is an important regulator of bone homeostasis and a mediator on inflammation-induced bone loss. $[14,30]$. FGF signalling activates or represses a network of transcriptional events in osteoblasts, like the induction of Sox 2 and the downregulation of Wnt signalling. In bone development, FGFs and Wnt have opposing roles: Wnt signals promote osteoblast differentiation and bone formation, whereas FGF signals inhibit osteoblast differentiation. Activation of Wnts protects from apoptosis and increases osteoblast differentiation. FGFs may thus increase apoptosis in osteoblasts via Sox2, by antagonizing Wnt signals.

Receptor activator of NF-KB ligand (RANKL) has an essential role in the development of osteoclasts. The extracellular portion is cleaved proteolytically to produce soluble RANKL, but the physiologic function of RANKL shedding has not yet been determined. Hikita et al. suggest that matrix metalloproteinase (MMP14) and a disintegrin and metalloproteinase (ADAM10) have strong RANKL shedding activity in bone marrow stromal cells and osteoblasts [19]. The in vivo role of RANKL shedding or skeletal homeostasis is currently unknown, because ADAM10-deficient animals have been reported to die prematurely, due to defects in heart and central nervous system development.

Other genes related to the immune system are modulated. This mechanism could be related to the biocompatibility process: indeed, the material has to be recognized as "self" by the immune system. CXCL10 is categorized functionally as a Th-1 chemokine. CXCL10 and CXCR3 may have important roles in leukocyte homing to inflamed tissues and in perpetuation of inflammation and tissue damage. The serum and tissue expressions of this protein are increased in autoimmune diseases, like rheumatoid arthritis, systemic lupus erythematosus, Sjögren syndrome, systemic sclerosis, idiopathic inflammatory myopathy. Lee et al. showed the CXCL10 pathogenic role in bone destruction via RANKL induction in inflamed synovial tissue of rheumatoid arthritis [20].

Serum levels of S100A12 reflect neutrophilic activation during synovial inflammation and the serum concentrations are correlated with synovial fluid levels and with disease activity in patients. The overexpression in a variety of auto-immune and autoinflammatory diseases and a number of proven proinflammatory effects of S100A12 in target cells highlight the relevance of this S100-protein in human inflammatory disorders. Initial—and at present unknownstimulus is supposed to be the starting signal for inflammatory arthritis and subsequently damage associated molecular patterns as endogenous molecules lead to an amplification and chronification of inflammation [20].

Starting this point, the ADAP, IKKbeta, FGFR2 and CXCL10 downregulation and PTHLH upregulation may decrease the inflammation and osteoclastogenesis and stimulate the osteoblasts.
MG63 cells are a cell line and not normal osteoblasts; however, this does not affect the meaning of these findings. Nevertheless, the advantages of using a cell line are related to the fact that the reproducibility of the data is higher, because there is no variability related to the studied source. Primary cell cultures provide a source of normal cells, but they also contain contaminating cells of different types and cells in variable differentiation states. Moreover, we have chosen to perform the experiment after 24 hours in order to get information on the early stages of stimulation. It is our knowledge, therefore, that more investigations with other osteoblast-like cell lines, primary cultures and different time points are needed in order to get a global understanding of the molecular events related to TT action.

This analysis can give a detailed description of the genetic effects of Trabecular Titanium on human osteoblastlike cells in vitro. Our data demonstrated that TT stimulates osteoblast proliferation, differentiation, and reduces osteoblast apoptosis. Moreover, TT limits the expression of genes related to osteoclastogenesis and inflammation and immunologic events. All these effects, therefore, contribute to improve biocompatibility and, consequently, the survival of a prosthetic implant. Finally, osteoblasts showed a similar behaviour when cultured on different TT porosities (data not shown).

In conclusion, Trabecular Titanium increases the transcription of some mRNAs, which enhance osteoblasts activity, while reducing osteoclastogenesis. No major adverse effects of TT on cells have been detected. These reported data encourage the clinical application of Trabecular Titanium to prosthetic devices.

\section{References}

[1] A. Wennerberg, T. Albrektsson, and B. Andersson, "An animal study of c.p. titanium screws with different surface topographies," Journal of Materials Science: Materials in Medicine, vol. 6, no. 5, pp. 302-309, 1995.

[2] L. H. Li, Y. M. Kong, H. W. Kim et al., "Improved biological performance of Ti implants due to surface modification by micro-arc oxidation," Biomaterials, vol. 25 , no. 14, pp. 28672875, 2004.

[3] C. Larsson, P. Thomsen, B. O. Aronsson et al., "Bone response to surface-modified titanium implants: studies on the early tissue response to machined and electropolished implants with different oxide thicknesses," Biomaterials, vol. 17, no. 6, pp. 605-616, 1996.

[4] F. Carinci, S. Volinia, F. Pezzetti, F. Francioso, L. Tosi, and A. Piattelli, "Titanium-cell interaction: analysis of cene expression profiling," Journal of Biomedical Materials Research-Part B Applied Biomaterials, vol. 66, no. 1, pp. 341-346, 2003.

[5] M. Keshmiri and T. Troczynski, "Apatite formation on $\mathrm{TiO}_{2}$ anatase microspheres," Journal of Non-Crystalline Solids, vol. 324, no. 3, pp. 289-294, 2003.

[6] D. Buser, "Titanium for dental applications. II. Implants with roughened surfaces," in Titanium in Medicine, D. M. Brunette, P. Tengvall, M. Textor, and P. Thomson, Eds., pp. 876-888, Springer, Berlin, Germany, 2001.

[7] R. Gapski, H. L. Wang, P. Mascarenhas, and N. P. Lang, "Critical review of immediate implant loading," Clinical Oral Implants Research, vol. 14, no. 5, pp. 515-527, 2003. 
[8] Z. Schwartz, J. Y. Martin, D. D. Dean, J. Simpson, D. L. Cochran, and B. D. Boyan, "Effect of titanium surface roughness on chondrocyte proliferation, matrix production, and differentiation depends on the state of cell maturation," Journal of Biomedical Materials Research, vol. 30, no. 2, pp. 145-155, 1996.

[9] J. Y. Martin, Z. Schwartz, T. W. Hummert et al., "Effect of titanium surface roughness on proliferation, differentiation, and protein synthesis of human osteoblast-like cells (MG63)," Journal of Biomedical Materials Research, vol. 29, no. 3, pp. 389-401, 1995.

[10] K. Gotfredsen, T. Berglundh, and J. Lindhe, "Anchorage of titanium implants with different surface characteristics: an experimental study in rabbits," Clinical Implant Dentistry and related Research, vol. 2, no. 3, pp. 120-128, 2000.

[11] B. R. Levine, S. Sporer, R. A. Poggie, C. J. Della Valle, and J. J. Jacobs, "Experimental and clinical performance of porous tantalum in orthopedic surgery," Biomaterials, vol. 27, no. 27, pp. 4671-4681, 2006.

[12] H. Matsuno, A. Yokoyama, F. Watari, M. Uo, and T. Kawasaki, "Biocompatibility and osteogenesis of refractory metal implants, titanium, hafnium, niobium, tantalum and rhenium," Biomaterials, vol. 22, no. 11, pp. 1253-1262, 2001.

[13] V. Sollazzo, F. Pezzetti, A. Palmieri, A. Girardi, F. Farinella, and F. Carinci, "Genetic effects of Trabecular Titanium ${ }^{\mathrm{TM}}$ on human osteoblast-like cells (MG-63): an in vitro study," Journal of Biomimetics, Biomaterials, and Tissue Engineering, vol. 9, no. 1, pp. 1-16, 2010.

[14] E. Schipani, B. Lanske, J. Hunzelman et al., "Targeted expression of constitutively active receptors for parathyroid hormone and parathyroid hormone-related peptide delays endochondral bone formation and rescues mice that lack parathyroid hormone-related peptide," Proceedings of the National Academy of Sciences of the United States of America, vol. 94, no. 25, pp. 13689-13694, 1997.

[15] E. J. Peterson, "The TCR ADAPts to integrin-mediated cell adhesion,” Immunological Reviews, vol. 192, pp. 113-121, 2003.

[16] M. G. Ruocco, S. Maeda, J. M. Park et al., "I $\kappa$ B kinase (IKK) $\beta$, but not IKK $\alpha$, is a critical mediator of osteoclast survival and is required for inflammation-induced bone loss," Journal of Experimental Medicine, vol. 201, no. 10, pp. 1677-1687, 2005.

[17] A. Raucci, P. Bellosta, R. Grassi, C. Basilico, and A. Mansukhani, "Osteoblast proliferation or differentiation is regulated by relative strengths of opposing signaling pathways," Journal of Cellular Physiology, vol. 215, no. 2, pp. 442-451, 2008.

[18] Y. G. Amaar, G. R. Thompson, T. A. Linkhart, S. T. Chen, D. J. Baylink, and S. Mohan, "Insulin-like growth factor-binding protein 5 (IGFBP-5) interacts with a four and a half LIM protein 2 (FHL2)," Journal of Biological Chemistry, vol. 277, no. 14, pp. 12053-12060, 2002.

[19] A. Hikita, I. Yana, H. Wakeyama et al., "Negative regulation of osteoclastogenesis by ectodomain shedding of receptor activator of NF- $\kappa$ B ligand," Journal of Biological Chemistry, vol. 281, no. 48, pp. 36846-36855, 2006.

[20] E. Y. Lee, Z. H. Lee, and Y. W. Song, "CXCL10 and autoimmune diseases," Autoimmunity Reviews, vol. 8, no. 5, pp. 379-383, 2009.

[21] D. J. Lovell and N. M. Ruth, "Pediatric clinical research," Current Opinion in Rheumatology, vol. 17, no. 3, pp. 265-270, 2005.
[22] H. Wittkowski, D. Foell, E. A. Klint et al., "Effects of intraarticular corticosteroids and anti-TNF therapy on neutrophil activation in rheumatoid arthritis," Annals of the Rheumatic Diseases, vol. 66, no. 8, pp. 1020-1025, 2007.

[23] J. R. Harrison, H. W. Woitge, and B. E. Kream, "Genetic approaches to determine the role of glucocorticoid signaling in osteoblasts," Endocrine, vol. 17, no. 1, pp. 37-42, 2002.

[24] D. A. Puleo and M. V. Thomas, "Implant Surfaces," Dental Clinics of North America, vol. 50, no. 3, pp. 323-338, 2006.

[25] U. Meyer, A. Büchter, H. P. Wiesmann, U. Joos, and D. B. Jones, "Basic reactions of osteoblasts on structured material surfaces," European Cells and Materials, vol. 9, pp. 39-49, 2005.

[26] B. D. Boyan, R. Batzer, and K. Kieswetter, "Titanium surface roughness alters responsiveness of MG63 osteoblast-like cells to 1 alpha,25-(OH)2D3," Journal of Biomedical Materials Research, vol. 39, pp. 77-85, 1998.

[27] K. Kieswetter, Z. Schwartz, D. D. Dean, and B. D. Boyan, "The role of implant surface characteristics in the healing of bone," Critical Reviews in Oral Biology and Medicine, vol. 7, no. 4, pp. 329-345, 1996.

[28] M. Bächle, F. Butz, U. Hübner, E. Bakalinis, and R. J. Kohal, "Behavior of CAL72 osteoblast-like cells cultured on zirconia ceramics with different surface topographies," Clinical Oral Implants Research, vol. 18, no. 1, pp. 53-59, 2007.

[29] G. J. Strewler, "The physiology of parathyroid hormonerelated protein," New England Journal of Medicine, vol. 342, no. 3, pp. 177-185, 2000.

[30] N. Kumagai, K. Ohno, R. Tameshige et al., "Induction of mouse c-src in RAW264 cells is dependent on AP- 1 and NF- $\kappa$ B and important for progression to multinucleated cell formation," Biochemical and Biophysical Research Communications, vol. 325, no. 3, pp. 758-768, 2004. 

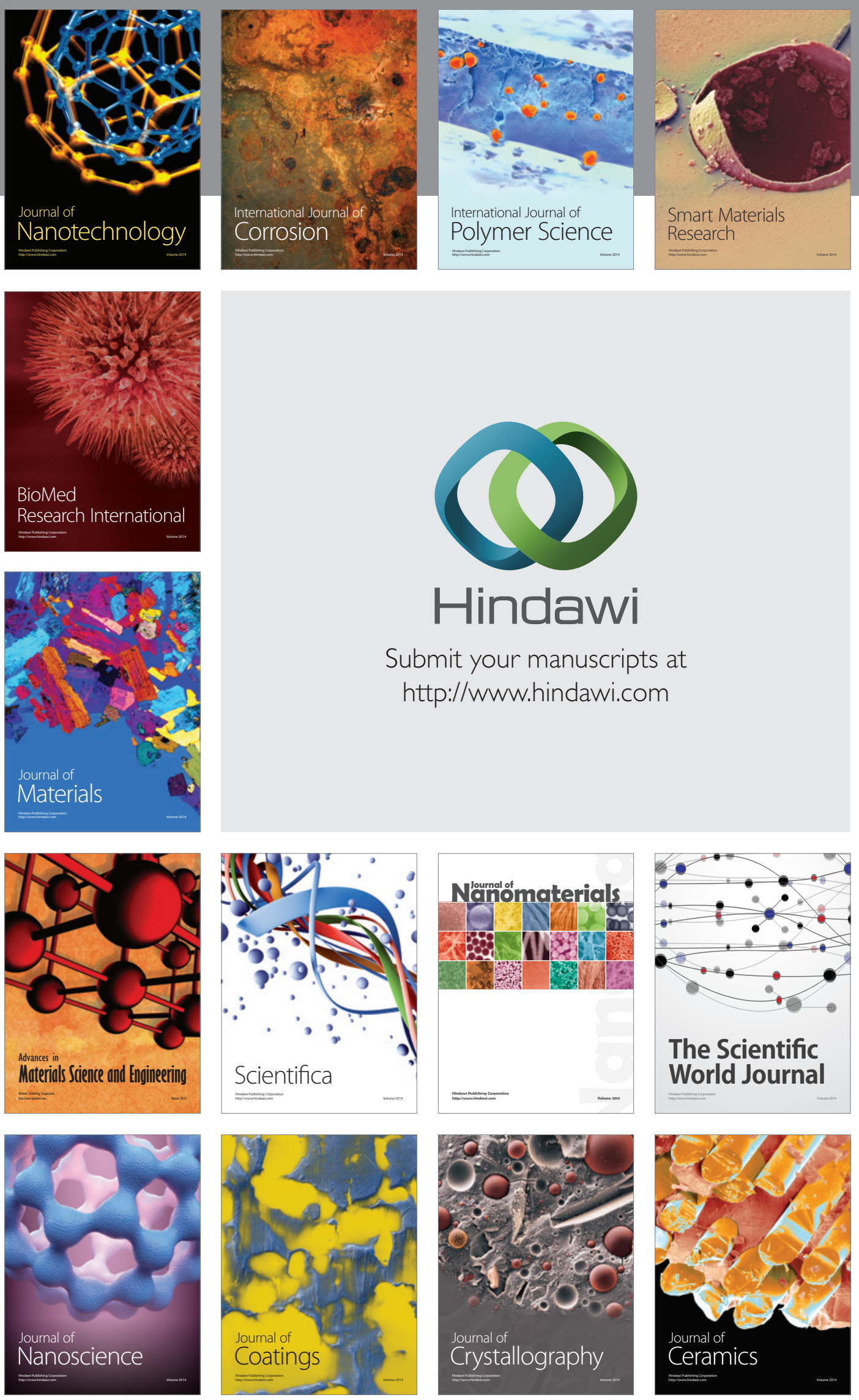

The Scientific World Journal

Submit your manuscripts at

http://www.hindawi.com

\section{World Journal}

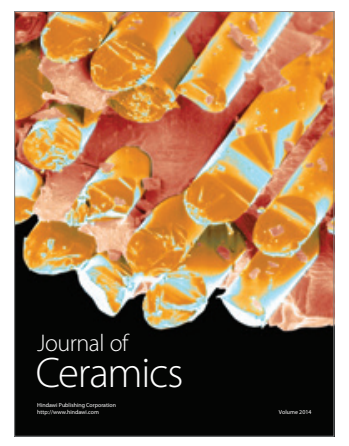

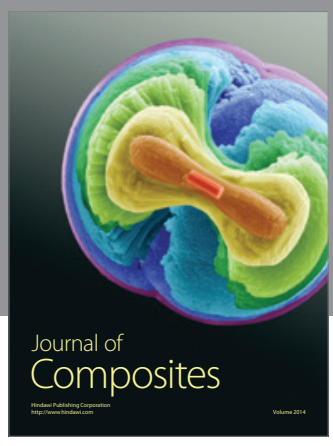
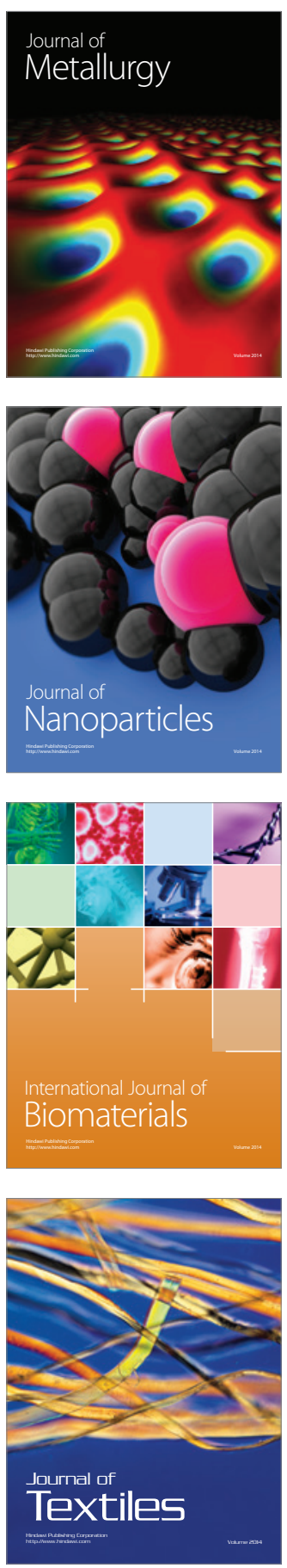